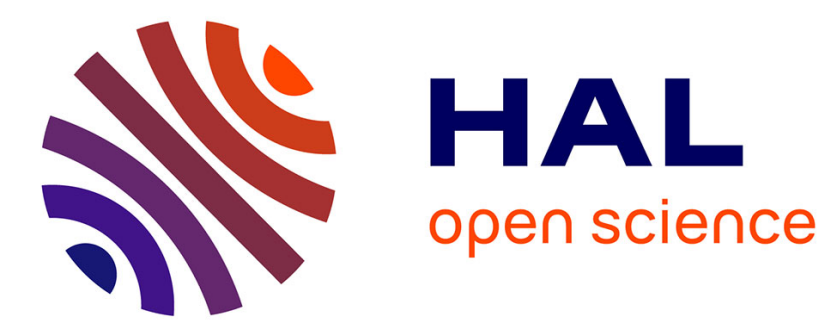

\title{
Les fondements philosophiques de la pensée de Carl Rogers
}

René Daval

\section{To cite this version:}

René Daval. Les fondements philosophiques de la pensée de Carl Rogers. Approche Centrée sur la Personne. Pratique et recherche, 2008, 8 (2), pp.5-20. 10.3917/acp.008.0005 . hal-02494964

\section{HAL Id: hal-02494964 \\ https://hal.univ-reims.fr/hal-02494964}

Submitted on 1 Mar 2020

HAL is a multi-disciplinary open access archive for the deposit and dissemination of scientific research documents, whether they are published or not. The documents may come from teaching and research institutions in France or abroad, or from public or private research centers.
L'archive ouverte pluridisciplinaire HAL, est destinée au dépôt et à la diffusion de documents scientifiques de niveau recherche, publiés ou non, émanant des établissements d'enseignement et de recherche français ou étrangers, des laboratoires publics ou privés. 


\section{Les fondements philosophiques de la pensée de Carl Rogers}

\section{René Daval}

\section{Université de Reims Champagne-Ardenne, CIRLEP}

Un préjugé tenace veut que l'œuvre de Rogers manque de profondeur et que ses concepts soient insuffisamment justifiés par l'analyse : il ne ferait que théoriser à partir du bon sens. La lecture d'un texte comme Psychothérapie et Relations humaines ${ }^{1}$ devrait suffire à elle seule à dissiper cette idée reçue, tant l'aspect méthodologique et expérimental est présent, et la théorie de la personnalité exposée est riche et fondée sur une longue expérience clinique. Mais je voudrais ici insister sur le lien qu'entretient la pensée de Rogers avec la philosophie, ou en tout cas, avec certaines philosophies. La clinique et la thérapie rogérienne croisent des philosophies et s'appuient sur elles, même si l'auteur reste souvent discret sur ses lectures et ses préférences philosophiques. Je vais $m^{\prime}$ attacher à montrer la continuité qui existe entre l'œuvre de Dewey et celle de Rogers, d'une part, et l'influence exercée sur celuici par l'existentialisme de Kierkegaard et le personnalisme de Buber, d'autre part. Suivra une réflexion sur la conception de la nature humaine qui est celle de Rogers, ce qui me conduira à étudier ses relations avec Freud. Je terminerai en marquant les liens qui existent entre le concept $d^{\prime}$ " empathie » et les philosophies allemandes de la fin du dix-neuvième siècle et du début du vingtième siècle.

\section{Éducation et démocratie}

Un préjugé tenace veut que l'œuvre de Rogers manque de profondeur et que ses concepts soient insuffisamment justifiés par l'analyse : il ne ferait que théoriser à partir du bon sens. La lecture d'un texte comme Psychothérapie et Relations humaines devrait suffire à elle seule à dissiper cette idée reçue, tant l'aspect méthodologique et expérimental est présent, et la théorie de la personnalité exposée est riche et fondée sur une longue expérience clinique. Mais je voudrais ici insister sur le lien qu'entretient la pensée de Rogers avec la philosophie, ou en tout cas, avec certaines philosophies. La clinique et la thérapie rogérienne croisent des philosophies et s'appuient sur elles, même si l'auteur reste souvent discret sur ses lectures et ses préférences philosophiques. Je vais $m^{\prime}$ attacher à montrer la continuité qui existe entre l'œuvre de Dewey et celle de Rogers, d'une part, et l'influence exercée sur celuici par l'existentialisme de Kierkegaard et le personnalisme de Buber, d'autre part. Suivra une réflexion sur la conception de la nature humaine qui est celle de Rogers, ce qui me conduira à étudier ses relations avec Freud. Je terminerai en marquant les liens qui existent entre le concept $d^{\prime}$ " " empathie » et les philosophies allemandes de la fin du dix-neuvième siècle et du début du vingtième siècle.

\section{Éducation et démocratie}

John Dewey et Carl Rogers partagent la même foi dans les idéaux démocratiques et pensent tous deux que l'éducation a pour fonction de développer la créativité inhérente en chaque personne, et que certaines institutions lorsqu'elles sont trop rigides, brident ou annihilent. Dewey a fortement insisté sur le fait que l'éducation a pour fonction d'aider le moi à se

\footnotetext{
1 N.d.e.: Rogers, C. R. \& Kinget, G. M., Psychothérapie et relations humaines, Louvain, Publications Universitaires, 1962 (épuisé).
} 
trouver lui-même. Le moi trouve son unité d'expression lorsqu'il ne poursuit plus qu'un seul but. L'idéal devient alors un motif d'action. Lorsqu'il fait de la psychothérapie, Rogers refuse d'être placé en position de maître face à son client, et il reproche souvent à la psychanalyse freudienne de mettre le client en situation d'infériorité par rapport à l'analyste. Le dispositif de face à face qu'il préfère, a cette fonction de mettre les deux protagonistes dans la même position. Le pédagogue Rogers, comme Dewey, veut que l'élève découvre par lui-même, développe son esprit d'investigation, sa curiosité, et ne soit pas soumis à un enseignement magistral qu'il subirait passivement. Les seules connaissances utiles sont celles qui modifient le comportement de la personne, et il est nécessaire que celle-ci les découvre elle-même et les fasse siennes. On ne peut communiquer ces connaissances aux autres, sous peine de les transformer en enseignement dont les résultats sont décevants. Rogers signale rejoindre sur ce point la pensée de Kierkegaard. Je reviendrai plus loin sur les liens que l'on peut établir entre le philosophe danois et le psychologue américain. Le pragmatiste John Dewey, comme Carl Rogers, fait appel à l'observation et à l'expérience. Il s'agit pour l'un comme pour l'autre de former une "personne" et non pas d'apprendre un comportement à un sujet d'expérimentation. John Dewey reprochait aux éducateurs de son temps de vouloir contraindre les enfants à effectuer des efforts, sans se préoccuper de ses intérêts, des capacités qui sont en lui et de l'esprit d'initiative dont il est capable. Dans L'école et l'enfant ${ }^{2}$, Dewey écrit : " quand on reconnaît l'existence chez l'enfant de certaines capacités qui ne demandent qu'à se développer, qui ont besoin qu'on agisse à partir d'elles pour en assurer l'efficacité et la discipline, nous avons une base solide pour construire ${ }^{3}$ ". L'effort naît sans trop grande contrainte parce que l'on veut rendre opératoires les capacités de l'enfant, et assurer la croissance et le plein épanouissement de celui-ci. L'éducateur s'assure l'assiduité de l'enfant puisque celui-ci se met au service de fins qui lui semblent valables. Effort et intérêt sont réconciliés, et l'effort n'est plus ressenti comme une corvée. Dewey et Rogers se retrouvent encore, eux qui insistent tous deux sur le fait que l'éducation est un processus dynamique qui passe par plusieurs phases et qui mobilise l'activité du moi dans le temps. Dewey insiste sur le lien qui existe entre l'intérêt, que l'éducateur doit susciter chez l'enfant, et notre être actif. Rogers, quant à lui, pense que le seul objectif éducatif est l'adaptabilité ${ }^{4}$. II ne faut pas transmettre un savoir figé, mais déclencher un processus qui se déroulera progressivement. II faut faciliter l'apprentissage. Développer la curiosité et le goût de la recherche, tout soumettre à la question, voilà comment il faut procéder. De même John Dewey jugeait que l'intérêt n'est pas un sentiment inerte à l'égard d'un objet. Comme Rogers accorde une grande importance à la place de l'affectivité dans le processus éducatif, Dewey notait qu'il n'y a pas d'intérêt sans émotion. L'individu est engagé dans une activité qui lui tient à cœur.

Il y a une philosophie de la vie chez Rogers comme chez Dewey : le premier parle de l'instinct d'accomplissement et de la tendance à l'actualisation que possède tout organisme vivant et qui le pousse à croître, à se développer et à réaliser ses potentiels, le second marque que le caractère actif de l'intérêt reflète les tendances et les impulsions de l'organisme vivant. Proche sur ce point de Bergson, Carl Rogers invoque l'élan vital qui conduit l'homme vers un développement plus complexe. L'Approche centrée sur la personne a pour fonction de libérer cet élan vital. Dewey, comme son ami et collègue G. H. Mead, estime que les

\footnotetext{
${ }^{2}$ Trad. L.S. Pidoux, Paris, Fabert, 2004, $1^{\text {re }}$ édition en français, Delachaux et Niestlé, 1913.

${ }^{3}$ P. 29.

${ }^{4}$ Voir par exemple L'approche centrée sur la personne, trad. H.G. Richon, Lausanne, Randin, 2001, p. 338.
} 
impulsions vont toujours dans une direction spécifique ${ }^{5}$. Le moi fait toujours quelque chose, il est toujours dans un état de tension. Il y a un caractère spontané et impulsif de l'activité de l'organisme. II y a toujours un objet interne ou externe qui nous intéresse. Dewey va jusqu'à écrire: "l'impulsion et le moi ne font qu'un; ils sont unis dans leur élan, dans leur mouvement ${ }^{6}$ ". L'objet n'a d'intérêt pour le moi que s'il est engagé dans l'activité de celui-ci comme c'est le cas dans le jeu. L'union qui existe entre l'apprentissage et les véritables intérêts de l'organisme est un thème que l'on retrouve dans les écrits de Rogers sur l'éducation.

Une autre conviction commune rapproche Carl Rogers et le pragmatisme et tout particulièrement celui de G. H. Mead et de John Dewey : celle selon laquelle il y a continuité entre la pédagogie et la formation de l'esprit citoyen : ce qui caractérise la démocratie, c'est l'égalité des droits de tous et la possibilité offerte de tenter de s'élever dans la hiérarchie sociale. L'enfant est un membre de la société et doit être traité comme tel à l'école, a souvent souligné Dewey. L'école a pour fonction d'assurer le maintien et l'amélioration de la vie sociale. Elle a un rôle moral à tenir. L'école doit s'occuper du développement de l'enfant considéré comme un tout. Elle doit apprendre à l'enfant à maîtriser l'ensemble de ses relations sociales. L'enfant sera un citoyen qui aura le droit de vote, mais il sera aussi le membre d'une famille et il aura sans doute la responsabilité d'élever à son tour des enfants et d'en faire des membres de la société. L'enfant sera un travailleur, jouant un rôle actif dans la production des richesses de la société. Il sera citoyen d'une commune et devra en ses lieu et place élever le niveau moral de la société. La théorie de la pédagogie active de Dewey (et de G. H. Mead, son ami et collègue de Chicago), tout comme la pédagogie et la théorie de la psychothérapie de Rogers poursuivent le même objectif: traiter l'autre comme une personne égale, à qui l'on doit donner les moyens de développer sa créativité. Le fameux principe rogérien en thérapie de "l'acceptation inconditionnelle du client " n'a pas d'autre but : il faut donner à celui-ci, en le mettant dans un climat de confiance affective, les moyens de se développer personnellement, d'actualiser les potentialités qui sont en lui. John Dewey et G. H. Mead poursuivent le même but. Dans Le développement de la personne ${ }^{7}$ Carl Rogers écrit : "j'en suis arrivé à croire que les seules connaissances qui puissent influencer le comportement d'un individu sont celles qu'il découvre lui-même et qu'il s'approprie ${ }^{8}$ ". Dewey, pour sa part, jugeait qu'il fallait s'appuyer sur les intérêts et les besoins de l'enfant pour qu'il trouve du plaisir à ses apprentissages. Il s'agit pour les deux penseurs d'aider une personne à devenir elle-même et à tirer profit des ressources qui existent en elle. Il faut se garder d'imposer une théorie ou un savoir, mais essayer en instaurant une relation authentique avec l'autre de l'aider à devenir lui-même. Un être qui parvient à l'existence authentique développe aussi des relations authentiques avec sa famille, ses amis, ses collègues de travail, ce qui profite à la société tout entière. À la fin de sa carrière dans son travail sur les groupes de rencontre, Rogers s'efforcera d'aider les membres du groupe à atteindre ce but ${ }^{9}$.

\footnotetext{
${ }^{5}$ G.H. Mead (1863-1932), philosophe américain, ami de Dewey et du sociologue Cooley, professeur à Chicago, est l'un des pragmatistes les plus importants et l'un des fondateurs de la psychologie sociale. Trois livres importants sont à retenir : La philosophie de l'action ; L'esprit, le soi et la société ; La philosophie du présent.

${ }^{6}$ Ibid., p. 31.

${ }^{7}$ Trad. E.L. Herbert, Dunod, Paris, 1966.

${ }^{8}$ Texte cité, p. 198.

${ }^{9}$ Les groupes de rencontre, trad. D. Le Bon, Paris, Dunod, 1973.
} 


\section{La conception de la science}

Dans un texte de la fin du Développement de la personne, Rogers souligne son accord avec la conception de la science qui était celle de Dewey : après avoir critiqué le néo-behaviorisme de Skinner, il ajoute : " nous nous trouvons ainsi en accord fondamental avec l'affirmation de John Dewey: "la science a fait son chemin en libérant, non en étouffant, les éléments de variation, d'invention, d'innovation, et de nouvelle création dans les individus ». La meilleure façon d'apprendre, estime Rogers pour sa part, est d'abandonner toute attitude défensive pour s'ouvrir au monde et à l'expérience. L'apprentissage passe par la meilleure compréhension de la signification réelle de l'expérience II faut laisser l'expérience guider le processus d'apprentissage dans une direction qui nous semble positive.

\section{Rogers et l'existentialisme}

Aider l'autre à être soi-même: Rogers n'est pas ici proche seulement du pragmatisme de Dewey et de G. H. Mead, mais il l'est aussi de Kierkegaard, dont il a souvent souligné l'influence sur son œuvre. Dans Le développement de la personne, on peut lire : " je crois que la meilleure façon d'exposer ce but de la vie, tel que je le vois dans mes rapports avec mes clients, est d'employer les mots de Soeren Kierkegaard : être vraiment soi-même ${ }^{10}$ ". Dans le Post-Scriptum aux Miettes Philosophiques d'où cette expression est issue, Kierkegaard souligne qu'il n'y a pas de système de l'existence et qu'on ne saurait penser l'individu en en faisant un élément d'un système comme le voulaient Hegel et ses disciples. L'existence se vit dans le secret, dans l'ironie, l'humour, le désespoir, dans la culpabilité, le sentiment du péché, dans l'espérance et pour certains dans l'expérience de la foi. C'est Heidegger et non pas Kierkegaard qui parlera d'existence inauthentique et d'existence authentique, mais l'idée est déjà chez le philosophe danois : c'est en assumant sa liberté que l'on devient une personne et non en multipliant des " expériences intéressantes " qui n'ont d'autre but que de nous permettre d'éviter le choix. II faut assumer sa responsabilité. L'existence véritable s'éprouve dans le choix. On ne saurait réduire le sujet existentiel à ses rôles sociaux, ni à quelque activité que ce soit. Être une personne, c'est vivre dans "l'isolement de la subjectivité ${ }^{11}$ ". II ne s'agit pas de prendre une importance digne de I'histoire du monde ou de la vérité du système. Il y a un secret de la subjectivité. Avec un vocabulaire moins philosophique, c'est à cela même que nous invite Carl Rogers. II ne s'agit pas pour le thérapeute de proposer un mode de vie, ni de donner des conseils, mais d'aider son client à découvrir ce qu'il veut vraiment, ce qui n'est possible que s'il comprend ce qu'il est vraiment. Dans son travail thérapeutique, Rogers ne cherche pas à établir un diagnostic, ni à donner des directives à ses patients. Ceux-ci s'éloignent progressivement de ce qu'ils ne sont pas. Les façades construites pendant l'éducation et la vie passée se fissurent. Le client commence à comprendre ce qu'il est. Ce "devenir soi-même ", pour reprendre une formule de Kierkegaard passe aussi par une confrontation avec les images que les autres ont de soi, et d'abord celles issues de l'éducation par les parents. Certains clients s'écartent de l'image longtemps incrustée en eux de ce qu'ils devraient être. La cure, finalement, conduit le client vers l'autonomie. La personne choisit les buts qu'elle veut atteindre dans sa vie, elle n'est plus simplement le reflet des désirs des autres. Rogers parle d'« autodirection

\footnotetext{
${ }^{10}$ Le développement de la personne, p. 124.

${ }^{11}$ Post-Scriptum aux Miettes Philosophiques, trad. P. H. Tisseau et E.-M. Jacquet Tisseau, Paris, éditions de l'Orante, 1977.
} 
responsable ${ }^{12}$ ». La psychothérapie se traduit par une plus grande flexibilité de la personnalité : le client se sent changer, se transformer, ne plus être prisonnier de schèmes de comportement rigides. Kierkegaard insiste sur le fait que l'individu est toujours en devenir et ne se dirige pas mécaniquement vers un but qu'il se serait fixé au préalable. Rogers est ici encore en phase avec le penseur danois : être soi-même, c'est devenir soimême. La personne s'ouvre davantage à sa propre expérience et devient plus tolérante visà-vis de parties d'elle-même qu'elle ne supportait pas auparavant. Cette transformation implique bien sûr de la part du thérapeute l'attitude d'acceptation inconditionnelle du client que Rogers préconise toujours.

On le voit, il s'agit pour Rogers d'apprendre à son client à assumer sa liberté, sa responsabilité d'être humain. Ici encore il rejoint Kierkegaard qui luttait tellement contre toutes les pensées totalisantes de son temps et notamment de l'idéalisme allemand avec Fichte et Hegel qui voulaient replacer l'individu dans la totalité de l'Histoire, dans l'organisation de l'État ou dans la marche de la raison dans l'Histoire. II faut replacer l'individu face à lui-même : à lui d'avoir le courage d'assumer sa responsabilité, à lui de choisir son mode d'exister. Pour devenir vraiment subjectif, analyse Kierkegaard dans le Post-Scriptum, il s'agit de savoir quelles données intellectuelles le sujet doit soumettre à la réflexion, de quelle objectivité il doit se défaire, quelle idée infinie il a de la valeur de ce changement. La subjectivité est effort personnel et total. Le devenir subjectif est un travail d'affranchissement. Rogers ne veut pas imposer ses vues au client qu'il a en thérapie, pas plus qu'il ne veut contraindre l'enfant à accepter passivement les valeurs et les enseignements du maître. Kierkegaard, comme Hamann quelques années avant lui, est très critique vis-à-vis des techniques éducatives de son temps ${ }^{13}$. Dans L'Alternative comme dans son Journal il regrette la pratique du par cœur et demande que l'on cherche à développer le moi de l'enfant. La quête de Kierkegaard s'exprime dans la maxime suivante : «trouver une vérité qui soit une vérité pour moi ". C'est à ce but que devrait tendre l'éducation qui, au lieu de cela propose des connaissances objectives sans explications à des enfants qui ne sont pas encore en âge de s'y intéresser. Rogers a dit et écrit qu'il y avait beaucoup d'aspects de la pensée de Kierkegaard qui lui étaient étrangers, et notamment son pessimisme quant à la nature humaine, mais il a très fortement marqué sa proximité avec lui. Tous deux jugent que la tâche essentielle est de développer la personne en soi et chez les autres. Rogers ne se réfère pas directement à la théorie des stades de l'existence de Kierkegaard, qui distinguait stade esthétique, éthique et religieux; il n'en reste pas moins que quand il décrit le processus thérapeutique, on retrouve certains aspects de la distinction kierkegaardienne. En effet, il s'agit pour le client de développer les capacités créatives qui sont en lui, et notamment de construire sa propre vie à partir de ses propres valeurs, ce qui est une caractéristique du stade éthique selon Kierkegaard, de savoir développer ses capacités organismiques et notamment sensorielles, ce qui renvoie au stade esthétique du philosophe danois, tandis que la vie spirituelle du client nous met en contact avec le stade religieux tel que le dépeint l'auteur de L'Alternative.

\section{Le Je et le Tu}

\footnotetext{
${ }^{12}$ Le développement de la personne, p. 128.

${ }^{13}$ Écrivain, essayiste et philosophe allemand, né en 1730, mort en 1788, surnommé le mage du nord, hostile à la pensée des Lumières, théoricien de l'humour et de l'ironie et penseur religieux.
} 
L'accent mis sur le concept de personne conduit Rogers à confronter sa pensée avec celle du philosophe Martin Buber, l'un des fondateurs du personnalisme. Rogers revient sans cesse sur la notion de personne: c'est ainsi qu'on peut lire dans la première partie de Le développement de la personne, intitulée " notes personnelles", l'affirmation suivante : " je parle en tant que personne dans un contexte d'expérience et d'apprentissage personnels ${ }^{14} »$. Rogers veut rencontrer l'autre en tant que personne. II veut " confirmer l'autre ", expression qu'il emprunte à Martin Buber ${ }^{15}$, comme il le signale lui-même $^{16}$, à la suite d'un dialogue tenu avec celui-ci à Ann Harbor dans le Michigan, le 18 avril 1957. Confirmer l'autre, c'est accepter toutes ses potentialités, c'est l'aider à laisser celles-ci se développer et s'épanouir. Confirmer l'autre, c'est faire confiance à sa créativité, et l'aider à la mettre en œuvre. Dans son ouvrage majeur Je et $\mathrm{Tu}^{17}$, Buber affirme que le monde est double pour l'homme, car l'attitude de l'homme est double en vertu de la dualité des mots principes qu'il peut prononcer. Au couple Je-Tu, base du langage, s'oppose cet autre couple de mots bases : Je-Cela. Ces mots fondent une existence. Dire Tu à l'autre, c'est s'adresser à lui en tant que personne, $c^{\prime}$ est refuser de le transformer en objet, c'est le respecter comme liberté, c'est, pour parler comme Kant, le traiter toujours comme une fin, et jamais comme un moyen. Buber distingue deux manières de traiter l'autre: comme un sujet, ou au contraire, comme un objet : "dire Tu, c'est n'avoir aucune chose pour objet. Car où il y a une chose, il y a une autre chose, chaque Cela confine à un autre Cela ${ }^{18}$ ". L'approche du monde par la science expérimentale ne nous livre que des Ceci et des Cela et nous rend impossible l'approche du mystère des êtres que seul le Tu peut nous faire entrevoir. Quand on dit à l'autre: Tu, il n'est plus pour nous une chose parmi les choses, il n'est pas un II ou Elle, limité par d'autres Ils ou Elles, il n'est plus un simple assemblage de qualités. En dehors de toute connexion, il est le Tu et toutes choses se reflètent dans sa lumière. Je ne découvre I'homme que j'appelle Tu dans aucun temps, et dans aucun lieu déterminé. Buber insiste : « tant que le ciel du Tu se déploie au-dessus de moi, les vents de la causalité s'accroupissent à mes talons et le tourbillon de la fatalité se fige ${ }^{19}$ ". On ne saurait avoir l'expérience du Tu, mais il vient à ma rencontre. C'est moi qui entre en relation immédiate avec lui. C'est une relation qui met en jeu l'être total. La relation se joue dans la réciprocité : je m'accomplis au contact du Tu, je deviens Je en disant Tu. La relation entre le Je et le Tu ne passe par la médiation d'aucun concept, ni d'aucune image. Il s'agit d'une relation entre deux présences vivantes.

\section{Rogers et le néo-béhaviourisme}

Rogers, pour sa part, craint que le développement des sciences du comportement ne puisse être employé pour aliéner la personnalité de l'homme, et milite pour une approche qui respecte la valeur de la personne. Carl Rogers insiste sur le fait que le psychothérapeute ne

\footnotetext{
${ }^{14}$ Ibid., p. 1.

${ }^{15}$ Le lecteur consultera avec profit l'article de C.-M. Leroy : " Martin Buber, précurseur de personnalisme », in ACP Pratique et recherche, $\mathrm{n}^{\circ} 1$, juin 2005, pp. 67-72.

${ }^{16}$ Le développement de la personne, p. 44.

${ }^{17}$ Trad. G. Bianquis, Paris, Aubier, 1969, édition allemande 1923.

${ }^{18}$ Je et Tu, p. 21.

${ }^{19}$ Je et Tu, p. 27.
} 
doit pas avoir en vue de prévoir les réactions de son patient pour pouvoir agir sur lui et faire de lui un être socialement acceptable, ce à quoi pourrait conduire une approche telle que celle de Skinner, mais doit vouloir l'épanouissement et le libre développement de celui-ci. II ne s'agit pas de contrôler le comportement humain, mais d'aider l'homme à devenir luimême.

La psychothérapie est une relation entre un Je et un $\mathrm{Tu}$, et non entre un Je et un II ou un Cela. C'est que, comme le fait remarquer Buber : " l'homme qui a la connaissance empirique des choses ne participe point au monde. La connaissance empirique se passe " en lui » et non entre lui et le monde. II n'y a pas de psychothérapie sans relations entre deux personnes, c'est-à-dire sans l'établissement d'un rapport entre un Je et un Tu. Dans la relation entre un Tu et un Je passe la vie. Rogers accepterait les termes des affirmations suivantes de Buber: "Le Tu est plus actif et il éprouve davantage que le Cela ne peut en avoir conscience. Aucune imposture n'a d'accès en ce lieu; c'est ici le berceau de la Vie $V_{\text {Veritable }}^{20}$ ". Nous ne pouvons avoir une connaissance empirique des hommes que nous appelons Tu. Nous sommes vraiment en relation avec lui dans le lieu sacré du mot fondamental Je-Tu. L'expérience, au contraire, m'éloigne du Tu. Nous ne savons rien de partiel au sujet du Tu. Adresser à l'autre le mot fondamental Je-Tu, c'est l'acte de mon être, c'est mon acte essentiel.

Carl Rogers développe une pensée analogue, lorsqu'il dit que le thérapeute doit avoir une relation de personne à personne avec son client, et non par exemple une relation de médecin à patient, ou d'expert à celui qui ne sait pas. Il s'agit pour le thérapeute de développer un processus de compréhension de soi chez son client, et de responsabilisation. II ne s'agit pas de trouver des solutions pour le client, et à sa place, mais de créer les conditions pour que le client puisse élaborer ses propres réponses aux questions existentielles qu'il se pose. Il faut que le client se sente libre de répondre à sa manière aux difficultés que la vie lui oppose. Rogers y insiste sans cesse : le psychologue « indique sans ambages que sa tâche est de créer un espace et une ambiance propices à l'approfondissement des problèmes, et à la mise à jour des relations. Mais [il] ne sousentend nullement qu'il serait de sa responsabilité d'apporter les réponses ${ }^{21}$ ". Le thérapeute encourage la libre expression des sentiments, que favorise son attitude de respect, d'ouverture à l'autre et d'écoute bienveillante. Accepter l'autre comme un Tu, c'est aussi accepter l'expression des sentiments négatifs qu'il peut avoir. Permettre l'expression des sentiments négatifs, c'est rendre possible l'acceptation par le client du fait que ces sentiments existent en lui, ce qui lui évite de les projeter sur les autres, ou de les dissimuler par des mécanismes de défense. Les sentiments négatifs acceptés, ce sont les sentiments positifs qui vont se manifester, comme l'amour, la sociabilité ou le désir de se conduire en adulte. De toute façon, le processus thérapeutique ne relève pas du jugement moral, et le thérapeute ne doit ni louer, ni blâmer son client. Il faut donner à la personne la possibilité de se comprendre telle qu'elle est. Le fait de s'accepter soi-même conduira la personne à un niveau supérieur d'intégration de sa personnalité. À la fin du processus thérapeutique, le client a de moins en moins peur, et devient de plus en plus autonome. Le client va pouvoir se passer du thérapeute. II a atteint la " congruence ", c'est-à-dire qu'il est devenu capable

\footnotetext{
${ }^{20}$ Idem.

${ }^{21}$ L'Approche centrée sur la personne, p. 95.
} 
d'affronter l'expérience vécue en pleine lucidité. La congruence permet la connaissance authentique de soi et des autres.

On le voit, Rogers et Buber ont en commun la conviction que la relation de personne à personne n'a rien à voir avec toute approche de l'autre considéré comme un objet à traiter ou à transformer à partir de notre propre conception de ce qu'il devrait faire. Mais il y a chez Buber l'affirmation que cette relation a une dimension de mystère religieux qu'on ne trouve pas chez Rogers.

\section{Rogers et la psychanalyse}

Venons-en alors à la conception de la nature humaine qui est celle de Carl Rogers. De nombreux critiques ont opposé sur ce point Freud et Rogers. Le premier verrait l'homme comme fondamentalement méchant, asocial et lubrique, tandis que le second, apparaissant ainsi comme le successeur de Rousseau, jugerait que l'homme est bon par nature, et deviendrait mauvais dans une société qui le corrompt ${ }^{22}$. Rogers juge que l'on ne peut faire de psychothérapie sans avoir une vue philosophique de ce qu'est l'homme, et il s'est souvent félicité de la confrontation que les critiques entreprennent de faire entre son travail et celui de Freud. Rogers souligne néanmoins que Rousseau n'a jamais exercé sur lui aucune influence directe, et qu'il ne l'a d'ailleurs pas lu. Il note avec force que son expérience de psychothérapeute ne lui a jamais fait observer que l'homme fut naturellement et foncièrement mauvais, asocial et destructeur. L'homme n'est pas une table rase, dépourvue de toute forme. Rogers ne croit pas non plus que l'homme serait naturellement parfait, puis qu'il serait corrompu par la société. L'homme a des caractéristiques spécifiques que l'on peut décrire en disant qu'il est positif, capable de se construire, capable de progresser, réaliste et fiable. Dans la relation d'aide empathique telle que Rogers la pratique, l'homme apparaît comme exprimant beaucoup de sentiments cruels ou meurtriers, comme agité de pulsions anormales ou de désirs bizarres. Mais à mesure qu'il découvre ses tendances en lui, qu'il devient lui-même et qu'il s'autonomise, l'homme révèle sa nature. On peut voir alors que l'homme est un membre de l'espèce humaine digne de confiance, qui tend par nature à se développer, à se différencier des autres, et à coopérer avec eux. L'individu humain passe de la dépendance à l'indépendance, réussit à autoréguler ses instincts, et il tend à sa survie et à son progrès comme à celui de l'espèce.

Rogers juge que « la véritable humanisation est un processus complexe qui fait de nous l'une des créatures les plus sensibles, les plus réactives, les plus créatives et les plus agréables de la planète ${ }^{23}$ ». Il est clair qu'en affirmant cela, Rogers s'oppose à la conception psychanalytique de l'homme, et notamment à celle que Freud expose dans Malaise dans la culture. II n'y a pas trace chez lui de l'affirmation, centrale dans les derniers écrits de Freud, et selon laquelle il y aurait en l'homme des pulsions de mort. II est loin aussi de Mélanie Klein, qui insiste sur les pulsions de destructivité de l'être humain et sur le noyau paranoïde de sa personnalité. Rogers oppose sa pensée à la psychanalyse freudienne, mais il est loin aussi de Jung quant à sa conception de la nature humaine. Jung estime en effet que

\footnotetext{
${ }^{22}$ L'Approche centrée sur la personne, p. 429.

${ }^{23}$ Ibid., p. 432.
} 
l'homme, lorsqu'il est le jouet de l'inconscient, individuel et surtout collectif peut se révéler un être dangereux, cruel et violent. Pour Rogers, au contraire, l'homme ne fait que développer les tendances qui sont en lui, et pourvu qu'il sache les reconnaître, il va aller dans le sens d'une plus grande sociabilité et d'une meilleure adaptation à la réalité tant naturelle que sociale. L'homme pour Rogers n'est pas foncièrement irrationnel. Le comportement humain est, au contraire, rationnel et vise les buts que notre organisme s'efforce d'atteindre. Ce sont seulement nos mécanismes de défense qui nous empêchent d'accéder à la rationalité. Notre organisme, libéré de ses défenses, tend naturellement à se développer harmonieusement.

S'opposant une fois encore à Freud, Rogers affirme : " en ce qui me concerne, je vois en l'homme une créature d'une admirable complexité, qui parfois tourne terriblement mal, mais dont les pulsions les plus profondes vont dans le sens du progrès, du sien comme de celui de ses congénères ${ }^{24}$ ॥. II faut cependant qu'il puisse jouir d'un climat rassurant pour pouvoir se développer vers cette intégration et cette harmonie supérieures, vers ce qu'il appelle la congruence. Pour Freud l'homme à l'état naturel est un loup pour ses semblables dont il veut faire l'objet de ses pulsions agressives ou sexuelles. Dans Malaise dans la Culture, il insiste longuement sur la nécessité que rencontre toute culture d'opposer des barrières aux pulsions, barrières que l'enfant va intérioriser et qui seront à l'origine de son surmoi. Pour Rogers, au contraire si l'homme peut poursuivre son développement naturellement, il va tendre sans excessive contrainte interne ou externe à l'harmonie avec lui-même et avec les autres. On peut de ce point de vue parler d'un optimisme de Rogers : s'il récuse la paternité de Rousseau et sa conception de la vie sociale comme pervertissant l'homme, il ne croit pas, contrairement à Freud, à Jung ou à M. Klein, que l'homme représente un danger pour ses semblables tant que ne se sont pas construits en lui des mécanismes psychiques ayant pour fonction de canaliser ses pulsions. Cet optimisme se retrouve dans sa théorie de l'éducation et, comme nous avons essayé de le montrer plus haut, le rapproche du pragmatisme de Dewey et de G. H. Mead.

\section{Empathie et sympathie}

Terminons en précisant les origines philosophiques de la notion d'empathie. On sait l'importance de l'empathie dans la thérapie comme dans les théories éducatives de Carl Rogers. II s'agit de comprendre une autre personne. II faut se garder de tout jugement de valeur, de toute évaluation, et essayer de saisir intellectuellement et avec des sentiments positifs les cadres de référence d'une autre personne. Rogers n'utilise pas le terme de compréhension en un sens banal : il faut comprendre ce terme en référence à la distinction traditionnelle depuis Dilthey entre "expliquer " et " comprendre ». Le philosophe Dilthey appelle " explication » la démarche de l'esprit à l'œuvre dans les sciences de la nature et qui consiste à relier entre eux des phénomènes par des lois causales mathématiquement exprimables. La compréhension, quant à elle, est la démarche des sciences de l'esprit, et d'abord de l'histoire, mais aussi de la psychologie, de la sociologie ou de l'économie. Il s'agit de reconstruire un sens à partir de la parole du sujet que l'on veut comprendre, du

\footnotetext{
${ }^{24}$ Ibid., p. 435.
} 
processus de décision qu'il met en œuvre dans ses décisions économiques ou des traces d'une culture que l'Histoire nous a léguées.

Rogers parle de compréhension au sens du Dilthey de l'Introduction à l'étude des sciences humaines $^{25}$. Jaspers avait repris cette distinction dans sa Psychopathologie générale (1923), et insistait sur le fait que la compréhension implique un rapport de proximité avec l'objet, et respecte l'individualité de celui-ci. Jaspers parlait d'" empathie " (Einfühlung). Les notions d'imitation et de contagion affective avaient été développées par Le Bon et Tarde en psychologie des foules, et par l'esthéticien allemand Theodor Lipps. Freud rapproche son concept d'identification de celui d'empathie, tout en maintenant une distinction entre eux. Jung, pour sa part réactualise la notion de " participation mystique » de Lucien Lévi-Bruhl. Mais l'empathie au sens de Rogers se rapproche aussi du concept de "sympathie " proposé par Max Scheler dans Nature et formes de la sympathie ${ }^{26}$. C'est, selon le phénoménologue, une disposition de la nature humaine qui a une valeur morale, car elle nous permet de nous mettre à la place d'autrui. Scheler distingue la sympathie de toutes les formes de contagion affective analysées par les psychologues des foules comme Gabriel Tarde ou Gustave Le Bon, tout comme de l'idée de "projection affective " et d' empathie " de l'esthéticien et éthicien allemand T. H. Lipps. C'est que pour Scheler, la sympathie implique que I'on ne fusionne pas avec l'autre, que l'on ait conscience de son altérité, et que l'on soit capable de connaître ce qu'il ressent. La sympathie n'est pas une fusion affective avec l'autre ou une identification à lui. Carl Rogers, de même, distingue l'empathie de la fusion à l'autre. L'empathie est un processus de connaissance et implique la conscience de l'altérité du client. Il s'agit de voir les choses comme l'autre les voit, sans jugement, sans prise de distance évaluative. II faut être capable de se mettre à la place de l'autre, en comprenant les événements de sa vie comme il les comprend. Il faut aussi avoir de l'affection pour l'autre. En aucun cas, en revanche il ne s'agit de ressentir la même chose que lui, de se projeter en lui, ou de revivre émotionnellement ses émotions. Rogers se demande : " aurai-je assez de finesse pour comprendre non seulement ce qu'il comprend clairement, mais aussi son nondit, ce qu'il ne perçoit lui-même que de manière trouble ou confuse ? Y a-t-il des limites à cette compréhension ? $^{27}$ ". La thérapie ne peut provoquer de changements positifs chez le client que si elle est fondée sur la compréhension empathique. Celle-ci permet au thérapeute de percevoir avec exactitude les sentiments éprouvés par le client, les significations qu'il donne à ses conduites, la manière dont il vit les situations dans lesquelles il se trouve placé. Le thérapeute doit faire partager à son client cette attitude d'ouverture aux autres et au monde et de disponibilité ${ }^{28}$. La capacité empathique permet de pénétrer suffisamment dans l'univers de l'autre pour éclairer les significations dont celui-ci est conscient, mais même celles dont il n'est pas encore conscient. Rogers prend grand soin de préciser, quand il définit l'empathie, le fait que le thérapeute doit rester lui-même et éviter de se projeter sur le client : il doit comprendre l'univers du client comme s'il était le sien, mais en restant conscient du « comme si ».

Dans L'Approche centrée sur la personne, on peut lire : " sentir la colère, la peur, l'embarras du client comme si c'étaient les siens, tout en restant à distance de cette colère, de cette

\footnotetext{
${ }^{25}$ 1901, trad. Sauzin, Paris, 1942.

${ }^{26} 1913$, deuxième édition modifiée en 1923.

${ }^{27}$ C. Rogers, L'approche centrée sur la personne, trad. H. G. Richon, Lausanne, Randin, 2001.

${ }^{28}$ L'approche centrée sur la personne, livre cité, p. 167.
} 
peur et de cet embarras : voilà la condition que nous essayons de décrire (en définissant ce qu'est l'empathie) ». Max Scheler jugeait que la participation affective (das Mitgefühl) était en tant que telle, tout comme toute forme de fusion affective, dépourvue de valeur morale parce qu'il n'y a plus de véritable intérêt pour l'autre, ni de prise en compte de celui-ci et que le sujet de cette participation reste en lui-même. Scheler parle en philosophe de l'éthique. Carl Rogers, psychothérapeute et aussi éducateur ne parle pas de valeur morale de l'empathie, mais il juge qu'elle n'a d'efficacité que si elle met en présence deux ou plusieurs personnes conscientes de leur individualité.

\section{Conclusion}

Si Rogers évite souvent d'employer un vocabulaire trop technique, et si son style est limpide et évite toute surcharge érudite, cela ne signifie pas pour autant que sa pensée est superficielle ou conduit à énoncer des banalités. J'ai essayé de montrer qu'il y a un arrièreplan philosophique que Rogers ne dévoile pas toujours dans ses textes, et qu'il rejoignait plusieurs grands penseurs sans toujours le signaler aux lecteurs. Si Kierkegaard et Buber sont des philosophes qu'il cite et avec lesquels il se reconnaît une fraternité de pensée, il évoque moins souvent John Dewey et le pragmatisme dont il est à certains égards proche, au moins dans sa réflexion sur l'éducation. Quant au débat sur l'explication et la compréhension, il ne pouvait l'ignorer car il se retrouve chez tous les théoriciens des sciences humaines de son époque. Je n'ai pas trouvé de référence directe à Scheler dans les textes de Rogers, mais l'emploi qu'il fait du terme même d'empathie conduit à trouver des convergences entre le phénoménologue allemand et lui. Rogers connaissait bien l'œuvre de Freud, et celui-ci connaissait les textes sur la contagion affective ainsi que Nature et Formes de la Sympathie de Scheler. Rogers, on le voit, n'était pas seulement un grand clinicien, mais sa pensée s'appuie sur des fondements philosophiques solides. La limpidité de son style ne doit pas nous induire en erreur : loin de s'en tenir au sens commun ses thèses sont fondées sur des analyses très rigoureuses de ce qu'est l'être humain, de ce que requiert la communication authentique, de la nature de l'empathie, du processus d'apprentissage et de transformation de la personnalité. 